\title{
NOTAS SOBRE VALENTÍN LETELIER Y LA FUNDACIÓN DEL INSTITUTO PEDAGÓGICO
}

Carlos Ruiz Schneider 


\section{CARLOS RUIZ SCHNEIDER}

Licenciado en Filosofía de la Universidad de Chile. Habilitación para dirigir Investigaciones, Universidad de Paris 8. Profesor titular, director del Departamento de Filosofía y profesor de la Facultad de Derecho de la Universidad de Chile. Áreas de interés: Filosofía política, historia del pensamiento político. 


\section{NOTAS SOBRE VALENTÍN LETELIER Y LA FUNDACIÓN DEL INSTITUTO PEDAGÓGICO}

La importancia de Valentín Letelier en la fundación del Instituto Pedagógico de la Universidad de Chile ha sido suficientemente documentada, en parte por él mismo, en sus escritos sobre el tema y en parte también por destacados historiadores de la educación en Chile ${ }^{1}$.

Lo que me propongo hacer en estas notas es revisar someramente el contexto intelectual más general en el que se insertan la acción y el discurso de Letelier sobre política educativa, sus ideas sobre la formación de los profesores, la Universidad y la educación, y, por último, algunas de sus observaciones sobre el Estado y el rol de este en la educación.

Para comprender bien las ideas de Letelier sobre educación, me parece necesario situarlas en el contexto de, por lo menos, dos debates políticos centrales en el período de fin de siglo. El primer debate es el que opone a los políticos e intelectuales republicanos cercanos al liberalismo, frente al radicalismo y los defensores de una presencia central de la Iglesia Católica en el campo cultural y educacional; una Iglesia que, entre tanto, ha ido acercándose cada vez más desde mediados del siglo XIX a posiciones ultramontanas².

En el campo republicano liberal destacan los aportes de José Victorino Lastarria, Miguel Luis Amunátegui, Diego Barros Arana y Valentín Letelier. En el campo conservador se destacan Abdón Cifuentes, Zorobabel Rodríguez y el obispo Joaquín Larraín Gandarillas, entre otros. Este conflicto político-religioso llega a su clímax en 1872, con

1. Sobre el papel de Letelier en la fundación del Instituto Pedagógico puede consultarse, del mismo Letelier, sobre todo su libro La lucha por la cultura (Santiago: Imprenta Barcelona, 1895); sobre los fundamentos teóricos que apoyan su creación, se puede consultar su Filosofía de la Educación (Santiago: Imprenta Cervantes, 1912). El papel de Letelier se encuentra también bien documentado por Rolando Mellafe y María Teresa González en el libro El Instituto Pedagógico de la Universidad de Chile (1889 -1981), Santiago, Departamento de Ciencias Históricas, Facultad de Filosofía y Humanidades, Universidad de Chile, 2007. Véase también, de Amanda Labarca, Historia de la enseñanza en Chile (1939); de Ricardo Krebs et al. Catolicismo y Laicismo (1981) y de Sol Serrano Universidad y nación: Chile en el siglo XIX (1994).

2. Véase sobre este punto, para el Caso de América Latina, de Tulio Halperin Donghi, Historia Contemporánea de América Latina (México: Siglo XXI, 1980). Sobre el caso francés, de mucha influencia en Letelier, véase Ozouf, Mona, L'Ecole, l’Eglise et la République, (Paris: Seuil, 1982). Y, de Marcel Gauchet, La religión dans la démocratie (Paris: Gallimard, 1998). Más recientemente, véase de Sophie Wahnich, "Defender la gratuidad escolar hoy (como ayer)" en Gutiérrez, C., Martin, A., Ruiz, C. y Vermeren, P. (comps.), Pasado y presente de la educación pública. Miradas desde Chile y Francia, Santiago: Catalonia, 2011. 
el ministro Cifuentes y la dictación de un decreto de libertad de enseñanza que establece, sobre todo, la libertad de exámenes para los colegios particulares. El conflicto escala con la destitución de Diego Barros Arana como rector del Instituto Nacional y luego con la renuncia de Abdón Cifuentes, que sigue a un voto de censura presentado por Guillermo Matta en la Cámara. Con la renuncia de Cifuentes y la derogación de los decretos de libertad de enseñanza, se consolida nuevamente un rol dirigente del Estado en la educación, a través de la supervigilancia de la Universidad de Chile sobre los exámenes y programas de la educación particular. Para la Revista Católica, la idea del "Estado Docente" implica un régimen tiránico que viola los derechos de las personas, las familias y la Iglesia. En el $N^{\circ}$ 1182, de 1873 de esta revista, podemos leer:

El Estado, he aquí la divinidad a que se rinde culto en nuestro siglo. Si se ha derribado a Dios de los altares... es para sustituir el culto del Estado al culto del verdadero Dios... nos espanta que hombres que se dicen liberales republicanos, miren, cual al bello ideal de la organización de la enseñanza, aquel en que el Estado la domina por completo. Este magisterio absoluto levantado en un país es la mayor de las monstruosidades i ningún sistema podría concebirse más a propósito para formar un pueblo de siervos... De veras la estatolatría recojitada por el liberalismo moderno es aún mucho más calculada i terrible que la que hacían dominar los déspotas de la antigüedad ${ }^{3}$. (p.186)

Frente a estos argumentos "liberales" de los conservadores, los intelectuales republicanos, como Letelier, llaman a tener en cuenta, en primer lugar, que aquí "no se traba la lucha entre el individuo i el Estado, como Spencer i los librecambistas lo suponen; trábase entre poder i poder, porque si nosotros (los radicales) tendemos a fortalecer el del Estado, ellos tienden a fortalecer el de la teocracia"4.

Cuando los partidarios de la teocracia, señala Letelier:

(...) piden, verbigracia, la llamada libertad de enseñanza, no tratan de conferir al hombre un nuevo derecho de enseñar libremente; este derecho, reconocido en nuestra Constitución, está anatematizado en el Syllabus. Lo que pretenden es que no enseñe el Estado para que enseñe sola la teocracia... reclaman también la libertad

3. En su vibrante "Discurso acerca de la libertad de enseñanza", pronunciado en el Senado en 1892, y que reproduce Mellafe, dice por su parte Abdón Cifuentes, entre otras cosas que: "El estanco intelectual de Chile confiado a una sola universidad, la universidad del Estado, tiene en sus manos el monopolio de las profesiones liberales, el monopolio de los grados universitarios...el monopolio de los exámenes anuales y por medio del monopolio de los planes de estudio, de los métodos de enseñanza, de los métodos y de cuanto se quiera; porque aunque la ley contempla la libertad de los métodos y de los textos, esa libertad es una mera burla, desde que en esta materia es una axioma que el dueño del examen es el dueño de la enseñanza... Nunca se vió despotismo semejante en ninguno de los pueblos de la tierra". Citado por Rolando Mellafe y María Teresa González en su libro El Instituto Pedagógico de la Universidad de Chile (1889-1981).

4. Letelier, V., La lucha por la cultura, Santiago: Imprenta Barcelona, 1985, pág. 6. 
relijiosa. Pero ¿acaso entienden por libertad relijiosa el derecho de de cada cual a profesar i enseñar la creencia de su amaño i fantasía? Evidentemente que no, porque esa libertad que es la que nosotros hemos sostenido... está condenada por el Syllabus...con el nombre de libertad relijiosa piden que reconozcamos a las manos muertas una existencia jurídica anterior a la autorización política e independiente del Estado; que desconceptuemos la justicia nacional i el principio de igualdad, confiriendo a los eclesiásticos un fuero especial...que so capa de doctrinar a los fieles, dejemos que el clero haga prédicas subversivas contra la Constitución, contra las leyes i contra las autoridades del Estado...5

En 1877, el ministro Amunátegui dicta un decreto que declara la admisibilidad de las mujeres para obtener títulos profesionales, y, en 1879, ve también la luz, poco después de que deja el Ministerio, la Ley Orgánica de Educación. Esta institucionaliza el rol docente y profesional de la Universidad de Chile, y declara la gratuidad de los estudios en las instituciones estatales desde la primaria a la universitaria.

Entretanto, la organización de la Iglesia, los intelectuales y los políticos católicos que buscan combatir a sus antagonistas usando sus "mismas armas", como lo dice don Carlos Casanueva, organizando el Partido Conservador, creando periódicos, revistas, asociaciones gremiales y hasta bancos. Esto tiene finalmente un fruto muy importante en 1888: la fundación de la Universidad Católica. Como lo expresa muy bien Ricardo Krebs en una monografía sobre el tema, la fundación de la Universidad "se inserta en el gran proceso de la redefinición de la actitud de la Iglesia Católica frente al mundo moderno"6. Sus propósitos son, al decir de su primer rector, Joaquín Larraín Gandarillas en la ceremonia de fundación en 1888, "(formar) no solo hombres ilustrados, sino también hombres morales y hombres cristianos"; y también "formar a la juventud que por su ilustración y condiciones sociales está llamada a influir de un modo más directo en los destinos de su patria" (pág. 27). Para Abdón Cifuentes, en una perspectiva mucho más realista, pero también conservadora, es preciso también fundar:

(...) la enseñanza social del pueblo; es preciso abrir nuevos y variados horizontes de sus vocaciones de actividad y trabajo...y es preciso multiplicar los medios de ganar la vida a los millares de jóvenes que serían perversos literatos, pero que pueden ser verdaderos genios en la industria. Menos compendios de enciclopedias ambulantes i más trabajo - dice con una retórica que le envidiaría Encina y que curiosamente es bastante común a las posiciones de la Iglesia en estos debates- menos retórica y más industria, menos sofistas y más ingenieros, menos teorías y más ciencias aplicadas: esto es lo que este país nuevo y laborioso necesita para acrecentar su riqueza, su prosperidad y su bienestar (pág. 27).

5. Letelier, V., "Ellos i nosotros o sea los liberales i los autoritarios", en La lucha por la cultura, Santiago: Imprenta Barcelona, 1985, págs. 7-8.

6. Krebs, R., "La Pontificia Universidad Católica de Chile 1888-1988", en Realidad Universitaria, № 6, Santiago: Centro de Estudios de la Realidad Contemporánea, 1988. 
En parte, como reacción a la fundación de la Universidad Católica, Letelier pronuncia el 16 de septiembre de 1888 en una sesión solemne de la Universidad de Chile, una de sus más importantes conferencias, titulada "El Estado y la educación": "Especialmente hoy -señala en esta conferencia- ante la tentativa que se hace para ocasionar en la sociedad chilena un fraccionamiento análogo al que trae convulsa i debilitada a la sociedad belga, el estado debe seguir más resueltamente que nunca las uniformes tradiciones de nuestra política docente" (1888, pág. 51). Recordándonos su postura permanente de que "el fin social de la enseñanza es la converjencia de todos los corazones a un mismo propósito i de todos los entendimientos a una misma fe" (pág. 47), Letelier sostiene que "un estado no puede ceder a ningún otro poder social la dirección superior de la enseñanza pública"... ya que bajo el respecto moral "Gobernar es educar i todo buen sistema de política es un verdadero sistema de educación, asi como todo sistema general de educación es un verdadero sistema político" (pág 44).

Asumiendo aquí de una manera modificada la doctrina positivista del "poder espiritual", que Comte y sus discípulos más religiosos encarnaban en los apóstoles de la religión de la Humanidad, Letelier subraya que es:

(...) doctrina esencialmente materialista, indigna de todo repúblico de espíritu superior, la de que el estado no debe curarse más que del cuerpo i del orden material de la sociedad. No señores! las tradiciones invariables de la política chilena no menos que los dictados de la sana filosofía nos enseñan que el estado tiene también cura de almas i de corazones, comoquiera que sumisión más elevada no es la de atender a la conservación del orden actual o material, sino la de atender al desenvolvimiento del orden eterno i moral. (p.44)

Y esta tarea espiritual es la que le corresponde, según nuestro autor, a la educación -en un sentido amplio- y especialmente a la instrucción universitaria.

Este último texto de Letelier me parece importante para caracterizar la profundidad del conflicto que opone en este momento, dentro de la sociedad chilena, a los partidarios ultramontanos de un poder educacional exclusivo de la Iglesia Católica, y los partidarios no menos extremos de una postura laicista radical.

Marcel Gauchet, en 1989, dentro de su libro Sobre la religión en la democracia, muestra que una postura similar a la de Letelier se desarrolla en Francia en los mismos años, tanto por Auguste Comte como por Charles Renouvier, quien había escrito en la revista La Critique Philosophique de 1872: "Sepamos bien que la separación de la Iglesia y el estado significa la organización del estado moral y educador"; y en 1876 que: "La supremacía del Estado es necesaria... Tiene (éste) cura de almas, tanto como toda Iglesia o comunidad, pero con un título más universal"7. El fundamento para esta supremacía, según Renouvier, es una moral independiente de la religión, cuyo modelo lo proporciona la teoría moral de Kant.

7. Textos de Renouvier citados por Marcel Gauchet en su libro La religión dans la démocratie (Paris: Gallimard, 1989), págs. 57-60. 
En un artículo reciente sobre "¿Qué significa secularismo?", publicado en su libro Dilemmas and connections. Ensayos selectos, de 2011, Charles Taylor -que se apoya para esto en parte en Gauchet- sostiene que gracias a la mayor moderación política de Jules Ferry y Jean Jaurès, entre otros, no es esta finalmente la concepción del laicismo que se impone en Francia, ni finalmente en Chile, habría que decir, donde una disputa como la del uso del velo por las adolescentes musulmanas es inimaginable ${ }^{8}$.

Este es, descrito muy someramente, uno de los debates que forman el contexto político e intelectual en el que Valentín Letelier, entre otros, puede darle finalmente curso a su proyecto de creación de un instituto universitario de formación de profesores de la educación secundaria.

El segundo debate, que se relaciona con el primero, tiene que ver con su defensa de la educación pública frente a los partidarios liberales e individualistas -pero también ultramontanos- de la educación privada. Aquí Letelier se opone sobre todo a los partidarios de Adam Smith, Herbert Spencer, John Stuart Mill y W. von Humboldt, y lo hace en función de una defensa del valor republicano y democrático de la igualdad.

En su Filosofía de la Educación, publicada en 1892, sostiene, por ejemplo, que: "las democracias tienen que dar preferencia a la educación pública porque la escuela común es una institución esencialmente democratizadora... forma la escuela una como república sujeta al régimen de la igualdad, república en que desaparecen las distinciones sociales de la fortuna i la sangre para no dejar subsistentes más que las de la virtud i el talento" (pág. 699). A lo que se agrega que "donde deja de ser pública, la enseñanza pierde su carácter democrático e igualitario, se convierte en simple adorno de las clases oligárquicas, i por el mismo hecho, se inhabilita para cumplir sus fines peculiares" (pág. 692).

Frente a los partidarios de una especie de laissez faire educativo, sostiene Letelier que la educación no es un bien de consumo: ni los profesores son productores de una industria ni los estudiantes son consumidores. Para muchos autores de nuestros días, afirma Letelier -y su pensamiento suena hoy muy actual- "esta industria debe ser tan libre que un ganapán cualquiera no tenga más trabas para establecer una escuela que para abrir una tienda. Nadie puede suponer que el público carezca de competencia para juzgar entre enseñanza i enseñanza, cuando la tiene para juzgar entre mercadería y mercadería" (pág. 703). Contra lo que aduce Letelier que la analogía es falsa, porque, a diferencia del consumo "el que no tiene instrucción alguna carece de competencia

8. Hay un interesante debate sobre el sentido del laicismo y la secularización en la filosofía actual. Tanto filósofos liberales como John Rawls o comunitarios como Charles Taylor se oponen a una concepción totalizante del laicismo. En el caso de Rawls, el rechazo se basa en su propuesta alternativa de un consenso superpuesto (overlapping consensus) que vale tanto para la filosofía como para la religión. En términos similares, Taylor propone una concepción de la secularización en términos de la protección de tres bienes similares a los propuestos por la Revolución francesa: "1. Nadie debe ser forzado en el dominio de la religión o creencias básicas. Esto es lo que a menudo se define como libertad religiosa, la que incluye, por cierto, la libertad de no creer... 2. Igualdad entre las personas de diferentes creencias básicas o fe; ninguna concepción del tipo de una Weltanshauung (religiosa o areligiosa) puede tener un estatus privilegiado como visión oficial del Estado y 3. Todas las familias espirituales deben ser escuchadas...". Véase Taylor, C., Dilemmas and Connections, Cambridge Mass: Harvard, 2011, pág. 309. 
para elejir entre enseñanza y enseñanza" (pág. 703). "Los economistas sostienen -agrega- que a virtud de la lei de la oferta i la demanda, cuando el estado no interviene, la iniciativa particular mejora la enseñanza i funda escuelas dondequiera se las necesita. Pero en el mayor la cultura i menor la necesidad, i no en las poblaciones más atrasadas, donde es mayor la necesidad, por ser menor la cultura. No son estas, entonces, empresas industriales, sujetas a la lei de la oferta i el pedido. Son empresas morales, sujetas a las necesidades de la cultura" (págs. 703-704)9 .

Enseñanza fundamentalmente pública y no confesional en todos sus niveles, he aquí la divisa educacional de Letelier, y es en función de este programa que podemos comprender mejor su profundo interés en la fundación del Instituto Pedagógico. Pero, para hacerlo, necesitamos aún echar una mirada un poco más precisa a sus ideas filosóficas y educativas.

\section{II}

Normalmente se adscribe, con razón, el pensamiento filosófico de Letelier a la escuela positivista, y esta visión es evidente en sus textos más importantes y en sus escritos educacionales más concretos, como cuando, por ejemplo, reflexiona sobre los fundamentos de los planes de estudios y propone cambios a fines de 1880 .

Aunque bastante alejado de muchas de las consecuencias conservadoras de las ideas de Auguste Comte, y más cercano a Littré que al positivismo más religioso, Letelier comparte, sin embargo, con el filósofo y matemático francés muchas de sus orientaciones importantes.

Comparte, en primer lugar, con casi todos los positivistas, una crítica muy radical del liberalismo individualista, a cuya influencia imputa consecuencias nefastas, tanto metafísicas como políticas. En realidad, como lo ha mostrado Robert Nisbet ${ }^{10}$, es a este individualismo al que todos los positivistas combaten bajo el nombre de metafísica y a él atribuyen la política anárquica salida de las "ideas de 1789", es decir de la Revolución francesa. Es con esta metafísica individualista de los derechos naturales con la que los positivistas contrastan la sociología, la ciencia de la sociedad, fundada por Comte. Con

9. Una analogía similar, y, a mi juicio, similarmente falsa, por las mismas razones que ya da Letelier en 1892, es la que propone Milton Friedman en su célebre artículo de 1958 sobre "El rol del gobierno en la educación", en el que se basaron las reformas impuestas por decretos de la dictadura militar en Chile. También para Friedman en ese artículo, en los intercambios educativos, los profesores son productores y los alumnos son consumidores. Como también lo predice Letelier, la desigualdad y la segregación han sido en Chile los resultados de entender a la educación de esa manera, como un bien de consumo. Por lo demás, podemos encontrar ideas similares en Adam Smith. Cita Letelier en la misma obra un pasaje de La riqueza de las naciones donde Smith había dicho "cuando el Estado interviene, la iniciativa individual no puede ostentar su potencia, sus esfuerzos i sus obras. Renuncien los poderes públicos a la tarea de administrar la enseñanza, i entonces las instituciones particulares la darán de mejor calidad i se multiplicarán en la justa medida en que cada pueblo la necesite" (Ibíd., págs. 706-707).

10. Véase sobre este tema de Robert Nisbet, La formación del pensamiento sociológico, Buenos Aires: Amorrortu, 1977. 
esta idea de una ciencia de la sociedad, se liga también la expectativa de construir una "política científica", la que puede ser compatible con un régimen político autoritario, como el porfiriato en México o la monarquía en Brasil.

Letelier acepta también esta idea de política científica, sin sus connotaciones autoritarias, y de aquí deriva la gran importancia que asigna a las instituciones que difunden y producen la ciencia, es decir, a las instituciones educacionales.

A su juicio, el principio aplicable a la sistematización de la enseñanza nacional "no puede ser otro sino aquel que simultáneamente satisfaga las dos necesidades supremas de nuestra época: la necesidad de trabajo inteligente i reproductivo i la necesidad de un credo común para todos los entendimientos i armonizador de todas las voluntades" (pág. 310). Esto significa organizar un sistema de enseñanza que "en medio de las múltiples divisiones, políticas, religiosas i sociales, sirva a modo de vínculo de unión entre todos los corazones i entre todos los entendimientos" (págs. 311-312). Ahora bien, continúa Letelier;

(...) no hai más que un sistema adornado de todos estos caracteres; no hai más que un sistema que, por lo mismo que no combate a ningún otro ni se opone a ningún otro, puede ser i es en realidad aceptado por todos: queremos hablar de la ciencia. Nadie duda de que uno más uno son dos, ni de que la tierra jira alrededor del sol, ni de que la electricidad produce el rayo, ni que la sensibilidad se transmite al cerebro por los hilos sensorios... estas verdades son las que a nuestro juicio... deben constituir la base fundamental de la enseñanza pública pagada por el Estado. (pág. 312)

La homogeneidad cultural y la cohesión social, estos son los grandes propósitos políticos de la educación para Letelier. Y ello solo puede ser el fruto de un sistema fundado en la enseñanza de la ciencia, de "la ciencia pura, nos dice, la ciencia que no profesa ni despierta odios, la ciencia sin hipótesis no comprobadas que alarman a muchas conciencias, la ciencia sin latines ni sectarismos que cinchan el cerebro i traban el libre vuelo del espíritu humano" (LC, La lucha por la cultura, pág. 313). Solo una educación de esta suerte, laica, formal y científica (pág. 752), puede ser objeto de la obligación escolar, en la instrucción general en sus distintos niveles ${ }^{11}$.

Aparte de estos objetivos, la enseñanza laica basada en una ciencia situada más allá de las hipótesis y las dudas, puede cumplir también otros propósitos, propiamente políticos, porque la educación tiene además una "tarea política, que forma en los pueblos cultos, la clase gobernante que ha de reemplazar a las antiguas, derribadas por la revolución i la cultura" (FE, pág. 141). En todas las épocas esta función es vital. Pero en la nuestra es imprescindible, porque vivimos, dice Letelier, "amagados no sólo por la revolución de los de abajo, sino también por la reacción de los de arriba; porque si los socialistas pretenden dar un carácter utilitario a todos los estudios, los clásicos no

11. La enseñanza universitaria debe ser pensada, según Letelier, de acuerdo a parámetros distintos, porque el tema fundamental de la Universidad no es difundir la ciencia sino producirla, lo que requiere plena libertad para los académicos. 
desesperan de darles carácter predominantemente literario, restableciendo la enseñanza obligatoria de las lenguas muertas" (FE, pág. sin referencia). Tenemos, pues, ante nosotros la tarea de formar una nueva clase dirigente, no podemos contentarnos ni con las tradicionales, ni estar de acuerdo con las clases revolucionarias. Y agrava la situación que tampoco el liberalismo -sin radicales transformaciones- sirva para estos propósitos, porque como lo asevera en el "Prólogo de la Lucha por la cultura": "Hoy, cuando todo Chile es liberal, este partido no tiene pueblo porque haciendo profesión de libre-cambio, se muestra insensible a las necesidades populares... de cierto esta no es una época de decadencia liberal, pero es una época de anarquía liberal" (VI-VII).

En la Filosofía de la Educación (FE), Letelier había identificado esta tarea con una tarea de la República:

Que la educación sea republicana bajo de la república; que fortifique en los corazones el respeto a las autoridades i a las leyes i el amor a la libertad i las instituciones; que reprima en la juventud con igual energía las tendencias reaccionarias contra los progresos realizados i las tendencias revolucionarias contra el orden establecido; que la forme en sentimientos de tolerancia, de concordia i de patriotismo: tales son las aspiraciones políticas que se deben realizar por medio de la enseñanza. (pág. 725)

Esta es la tarea política del partido político en el que milita nuestro autor, el Partido Radical, como lo dice en un notable artículo sobre" Los pobres", publicado en el diario La Ley, el $1^{\circ}$ de enero de 1896, en donde podemos leer:

(...) por su posición intermedia entre las clases más egoístas i las más desvalidas, a mi juicio es el partido radical el llamado a salvar la sociedad chilena de las tremendas convulsiones que ajitan a la sociedad europea. Proveer a las necesidades de los desvalidos es remover la causa del descontento, es acabar con el socialismo revolucionario, es hacer política científicamente conservadora.

No es posible fundar esta política, nos dice Letelier, sobre la base endeble y no científica del liberalismo actual. En La lucha por la cultura había afirmado que "debemos estirpar de nuestro espíritu la preocupación metafísica i revolucionaria que supone ser la libertad una panacea propia a curar todas las enfermedades del organismo social". Piensa que nada ha colaborado más en su tiempo al avasallamiento de muchos pueblos "que la absoluta confianza de los liberales en el régimen de la libertad... convencidos de que ella tiene ciertas virtudes ocultas para curar todos los males, han solido renunciar a toda cooperación de la parte del Estado; e imaginándola dotada de una vida propia, inmortal e independiente de las condiciones sociales han solido anular los poderes llamados a protejerla i han dejado imprudentemente desarrollarse fuerzas reaccionarias..." (LC, págs. 22-23). El fin de la política -nos dice, también en LC-"no es la libertad, no es la autoridad ni es principio alguno de carácter abstracto, sino que es el de satisfacer las necesidades sociales para procurar el perfeccionamiento del hombre i el desarrollo de la sociedad" (LC, pág. 31). 
Veamos ahora de más cerca el pensamiento de Letelier acerca de los profesores y la formación universitaria de los mismos, especialmente de los profesores secundarios, para analizar sus ideas sobre la creación del Instituto Pedagógico.

La impresión que deja la revisión de los textos sobre el tema, es que para comprender plenamente el esfuerzo y el interés de Letelier por la formación universitaria de los profesores y la fundación del Instituto Pedagógico, es vital considerar el contexto político e intelectual que acabamos de evocar.

Y en primer lugar, me parece que tenemos que considerar, en este punto, un acontecimiento político-cultural que tiene lugar un año antes de la fundación del Instituto en abril de 1889: me refiero a la fundación de la Universidad Católica en julio de 1888.

En un ensayo que citábamos más arriba, y en el que Letelier se refiere a la Universidad Católica, insiste, por ejemplo, en que en ese momento el Estado debe reservar:

(...) esclusivamente para sí todas aquellas prerrogativas jurídicas, como la colación de grados, que sólo en virtud de una delegación espresa de su soberanía, pueden ser ejercidas por autoridades estrañas. Cuídese de ceder a otras manos fuerzas i armas que juntamente servirían para combatirlo a él i para ahondar las divisiones de la sociedad chilena. Contrarreste decididamente la influencia sectaria de una enseñanza que propende derechamente a reaccionar contra el desarrollo de nuestra cultura social, con la influencia moderadora de una enseñanza puramente científica, que no vulnera los fueros de ninguna conciencia. I sobre todo, ponga los institutos de instrucción pública en condiciones de superioridad tal, que todo padre de familia se sienta inclinado a preferirlos para la educación de sus hijos. (LC., pág. 51)

Sin embargo, Letelier piensa que para cumplir su misión, la Universidad de Chile adolece de graves deficiencias. Una de las principales, sostiene, es que:

(...) es sabido que la más noble de sus facultades, la llamada a ser el verdadero seminario del profesorado secundario i a servir en este carácter de lazo de relación entre el instituto superior que crea la ciencia i los institutos secundarios que la difunden, la facultad de filosofía y humanidades, en fin, no existe sino en el papel i en el nombre. Es también sabido que por esta misma causa, aquellas ramas de la ciencia que atañen al orden moral, que son las que mas realzan su aplicabilidad social i las que la elevan a la dignidad de una filosofía general, no están representadas por una sola cátedra.

Afortunadamente, prosigue, Letelier, en el momento actual "dos nuevos proyectos que se trata de llevar a cabo, el de un curso de administración i política i el de un seminario pedagógico, están destinados a llenar en gran parte esos vacíos” (Ibíd., pág. 48).

Piensa Letelier que en "el árbol de la enseñanza", la raíz es la Universidad y "la flor es la escuela", por lo cual no habrá una buena enseñanza primaria y universal sin enseñanza universitaria (FE, pág. 503). Esta generación universitaria de "todas las enseñanzas 
nacionales es, por consiguiente un fenómeno social" que propende a abarcar todas las ciencias para formar una sola ciencia general, y por ello "propende también a rejir todas las enseñanzas para formar una sola enseñanza nacional" (FE, pág. 505). Por esto, con Victor Cousin, piensa Letelier que "la universidad es la instrucción pública entera, pero deja de serlo desde el momento en que se sustrae a su imperio un solo liceo, una sola escuela i se convierte en un instituto particular de simple enseñanza" (FE, págs. 505-506).

Continuando esta reflexión, agrega Letelier que la enseñanza universitaria sería "una función que no ejercería mayor influjo en la formación del intelecto nacional si no fuese porque directa o indirectamente da vida a un cuerpo de funcionarios que difunde su espíritu hasta los últimos rincones de la sociedad: quiero hablar del personal docente" (FE, pág. 517).

Por esto piensa nuestro autor que es vital que la Universidad no abandone esta tarea, cuestión que está sucediendo en muchos países, fundando escuelas normales, seminarios pedagógicos o institutos pedagógicos, como será el caso en Chile. Desde su perspectiva, "el deber más trascendental del profesorado en las sociedades cultas es, como lo hemos visto antes, restablecer la unidad del intelecto; i no puede acercarse a la realización de este lejano ideal sino recibiendo directa o indirectamente de unas solas manos, de manos de la universidad, a la vez que la investidura del magisterio, la comunión de una misma verdad" (FE, pág. 521).

Piensa Letelier que de las aseveraciones que anteceden, se infiere que "no es la enseñanza la principal función de las universidades... es la de jenerar una clase selectísima de intelectuales que a pesar de las tendencias utilitarias del medio ambiente, mantenga encendido el culto desinteresado de la ciencia, de las letras i de las artes, adquiera el espíritu i el hábito de las investigación i sirva de cuna i origen al personal docente de los Estados" (FE, pág. 538).

Necesita la Universidad de libertad, pero la libertad que necesita -dice Letelier- "no es aquella libertad reaccionaria que los economistas católicos reclaman i que prácticamente se reduce a suprimir la injerencia del Estado en la enseñanza...la libertad que la ciencia necesita para desarrollarse es la libertad de enseñanza, de investigación i de pensamiento; i esta libertad no es incompatible...con la sujeción administrativa de la universidad a la autoridad política" (FE, pág. 526)12.

Y Letelier concluye estas reflexiones con la reafirmación de la formación universitaria de los profesores, ya que "el poder espiritual que en las sociedades cultas propende espontáneamente a desarrollarse es el del profesorado, o sea, el de aquellos que, consagrados a las tareas de la enseñanza i del estudio, se ocupan o en propagar o en descubrir las verdades de la ciencia" (FE, pág. 553).

12. En la FE Letelier comenta muy críticamente afirmaciones del obispo Larraín Gandarillas que unen curiosamente en el siglo XIX al ultramontanismo con el liberalismo. "El Estado -dice Larraín Gandarillas- no debe poner obstáculos a la industria privada para fundar establecimientos de educación, ni hacer competencia a los ya establecidos, revistiendo a los fiscales de privilegios que dificulten la concurrencia" (FE, págs. 702-703). "Cuando uno oye hablar así -comenta Letelier- se imajina habérselas, no con el abnegado rector del Seminario episcopal de Santiago, sino con el interesado empresario de alguna fábrica de tejidos" (FE, pág. 703). 
El análisis de los escritos hasta aquí citados revela, me parece, que el objeto central en la disputa ente laicistas y católicos ultramontanos, es la formación de las clases dirigentes de la sociedad chilena. Y conseguir consolidar una hegemonía laica sobre los grupos dirigentes, a través de la constitución de la Universidad de Chile, el Instituto Pedagógico y los profesores secundarios formados por este, es el objetivo político central que subyace a fundación del Instituto Pedagógico.

Por cierto, esta disputa no es solo una disputa por el poder y la hegemonía. Ambas posiciones implican visiones muy diferentes de la sociedad chilena, que no se reducen a esta oposición evidente entre catolicismo ultramontano y laicismo.

Como lo hemos mostrado, en el caso de Letelier, el laicismo en educación tiene un claro compromiso republicano y, dentro de ciertos límites, igualitario y democrático. Este compromiso social se expresa en la adscripción política de muchos de sus defensores al Partido Radical, un movimiento político que busca expresar a nuevos grupos sociales en el Estado, para producir un cambio en la hegemonía tradicional en Chile. Por su parte la visión ultramontana, con su compromiso con el liberalismo del laissez faire, no es en absoluto opuesto a la desigualdad social. El compromiso del conservadurismo católico con las clases dirigentes más tradicionales es mucho más claro. Estas posiciones de los católicos conservadores ante la igualdad y la desigualdad en la sociedad se expresan, por ejemplo, en el discurso de incorporación a la Facultad de Filosofía y Humanidades de la Universidad de Chile, del canónigo y Decano de la Facultad de Teología de la Universidad, Joaquín Larraín Gandarillas en 1863. Dice en su discurso Larraín Gandarillas, que se debiera a un tiempo fomentar y restringir los estudios del curso de humanidades:

(...) fomentarlo, mejorando la enseñanza; restringirlo, disminuyendo el número de los que lo abrazan. Las haría obligatorias como preparación para las carreras científicas, sobre todo para la del foro i la eclesiástica, de las que han de salir los ciudadanos que tienen que ejercer más influencia en el Estado. No las haría mui accesibles a las clases bajas de la sociedad. ¿Qué gana el país con que los hijos de los campesinos i de los artesanos abandonen la condición en la que los ha colocado la providencia, para convertirlos las más de las veces en ociosos pedantes... que colocados en una posición falsa terminan por aborrecer la sociedad? ${ }^{13}$

No nos sorprenderá, entonces, la entusiasta y comprometida participación de Valentín Letelier en todos los pasos previos a la fundación del Instituto Pedagógico, en la última etapa, en íntima colaboración con Claudio Matte. Y esto, durante el Gobierno de Balmaceda, del que Letelier es ferviente opositor. Defiende este logro del Gobierno de Balmaceda después de la derrota de este, en contra de quienes quieren confundir sus logros y rechazarlos en conjunto. Pero también en contra de quienes en la misma Universidad de Chile y el Consejo de Instrucción Pública se oponen a su creación ${ }^{14}$.

13. Larraín Gandarillas, J., Anales de la Universidad de Chile, 1863.

14. Sobre las críticas y la oposición a la creación del Instituto, véase, de Valentín Letelier, "El Instituto Pedagógico. Sus precursores", en La lucha por la cultura, op. cit. 
El modelo institucional de la fundación del Instituto Pedagógico es la Escuela Normal Superior de París. Pero para la constitución de su primer personal docente y directivo, Matte y Letelier proponen la contratación de un selecto grupo de profesores alemanes, en disciplinas como la filosofía y la pedagogía, las matemáticas, las ciencias naturales, la historia y geografía y la filología. Estas contrataciones se basan en el íntimo convencimiento de Matte y Letelier de que ninguno de los más destacados académicos e investigadores nacionales es un conocedor de la ciencia pedagógica, de la didáctica, en la que Alemania en la época se destaca muy especialmente, desde la enseñanza parvularia, con Fröbel, hasta los seminarios especializados en la formación de profesores. De aquí esta peculiar combinación de una institución marcadamente republicana y estatal, como la École Normale Supérieure de París y la ciencia pedagógica del Reich alemán, que dará su sello a nuestro Instituto Pedagógico por muchos años, asegurándole su alta calidad en la formación docente. 